\title{
Contribuciones de enfermería a la anestesiología en Costa Rica ${ }^{1}$
}

Institución: Universidad de Costa Rica

Alba Carranza Ramírez ${ }^{2}$

\section{COMO CITAR}

Moreira, A. y Murillo, P. (2016). Aportes de la enfermería a la anestesiología en Costa Rica. Rev. Enfermería Actual de Costa Rica, 30, 1-12. DOI: http://dx.doi.org/10.15517/revenf.v0i30.22209

\section{RESUMEN}

Introducción. En el presente artículo se presentan los resultados de un estudio que cuyo objetivo fue identificar la participación de la enfermería en el desarrollo de la anestesiología en Costa Rica.

Método. Se aplicó la metodología de la investigación histórica para determinar los sucesos ocurridos en el pasado relacionados con el desarrollo de la anestesia, así como las contribuciones de enfermería. Se analizó fuentes primarias y secundarias que fueron sistematizadas, valoradas, ordenadas y sintetizadas. Se aplicó la técnica de grupo focal y entrevistas en profundidad a 23 enfermeras (os) técnicos en anestesia que laboran actualmente y a las enfermeras (os) pensionados.

Resultados. Durante más de 90 años la administración de anestesia a las personas fue llevada a cabo por enfermeras (os), no obstante, la formación de enfermería en anestesia fue suspendida en 1981.

Conclusión. El estudio determinó que este personal tenía una preparación formal dada por el doctor Sotela que luego fue ampliada a año y medio por la Escuela de Medicina de la Universidad de Costa Rica, institución de la que egresaban como técnicos en anestesia.

Palabras clave: Enfermería, historia, anestesia

\footnotetext{
${ }^{1}$ Fecha de recepción: 26 de agosto 2015

Fecha de aceptación: 2 de diciembre 2015

${ }^{2}$ Enfermera. Universidad de Costa Rica. Costa Rica. Correo electrónico: alircara@gmail.com
} 


\section{Contribution nursing to the anesthesiology in Costa Rica ${ }^{1}$}

Institution: University of Costa Rica

Alba Carranza Ramírez ${ }^{2}$

\section{CITED:}

Carranza, A. (2016). Contribution nursing to the anesthesiology in Costa Rica. Rev. Enfermería Actual de Costa Rica, 30, 1-13. DOI: http://dx.doi.org/10.15517/revenf.v0i30.22209

Introduction. In this article the results of a study that aimed to identify the nursing involvement in the development of anesthesiology in Costa Rica are presented.

Method. Historical research methodology is applied to determine the events related to the development of anesthesia as well as the contributions of nursing. The primary and secondary sources were systematized, evaluated, ordered and synthesized. The technique of focal group and interviews in depth with 23 nurses anesthesia technicians that currently working and pensioners was applied.

Result. During more than 90 years staff of the discipline conducted the administration of anesthesia to people and training in anesthesia nursing was suspended in 1981.

Conclusion. The study found that the staff had formal training given by Dr. Sotela which was later extended to one and a half by the School of Medicine of the University of Costa Rica, an institution that were teaching as technicians in anesthesia.

Keywords. Anesthesiology, history, nursing.

\footnotetext{
${ }^{1}$ Date of receipt: August 26, 2015

Date of acceptance: December 2, 2015

${ }^{2}$ Nurse. Professor of the University of Costa Rica. Costa Rica. E mail: alircara@gmail.com
} 


\section{Revista Electrónica Enfermeria Actual en costa Rica}

\section{INTRODUCCIÓN}

La enfermedad es una situación que ha acompañado al ser humano desde la antigüedad, siendo el dolor un síntoma que las acompaña; por lo que la anestesia ha proporcionado, a través de sus diversos métodos y aplicación de remedios contra el dolor, grandes contribuciones a la medicina. De igual forma, enfermería ha contribuido a disminuir el dolor de las personas mediante su protagonismo en la anestesiología, desde la preanestesia, durante el proceso perioperatorio, en el postoperatorio y dando apoyo ante la presencia del dolor crónico. La enfermera de anestesia, "es una enfermera que está cualificada y especializada en el ejercicio de la práctica anestésica” (González, Mugabure y Uria, 2010, p. 5)

Por otro lado, es importante acotar que la anestesiología data desde las civilizaciones antiguas con diferentes prácticas, muchas de las cuales ponían en riesgo la vida de las personas que eran sometidas a este tipo de intervenciones. Ejemplo de ello es lo referido por Higgins (s.f.)

3000 A.C. los asirios conocían un método eficaz para causar "anestesia", aunque no exento de peligro, comprimiendo la carótida a nivel del cuello con la consiguiente isquemia cerebral y la aparición de un estado comatoso lo cual era aprovechado para la cirugía. (s.p)

Los métodos utilizados por la anestesiología han evolucionado, identificándose sustancias con efectos anestésicos, dosis para las mismas, entre otros aspectos. Se ha determinado que a través de la historia son los médicos y las enfermeras (os) los que se han encargado de llevar a cabo las intervenciones en anestesia Es por ello, que este documento presenta la historia de las intervenciones en anestesia por parte de enfermería, específicamente en Costa Rica.

Debido a las pocas publicaciones relacionadas con la participación de enfermería en esta área, a excepción de manuales de actualización como el presentado por González y otras (2010), el cual menciona la participación activa de enfermería en Estados Unidos y en la mayoría de países europeos, llevó a la necesidad de investigar la temática con el propósito de conocer las contribuciones de enfermería en el desarrollo de la anestesia en Costa Rica.

Es importante destacar que la participación de enfermería en esta área de atención de la salud ha sido una de las más antiguas del mundo, particularmente en Estados Unidos, país en el que, desde aproximadamente en el año 1800, los médicos reclutaron y adiestraron enfermeras para realizar el cuidado de anestesia de los pacientes, (Hamric, Spross y Hanson (1996), mencionados por Vélez, 1996)

Es por lo anterior, que este documento invita a reflexionar desde la perspectiva del desarrollo histórico de la disciplina enfermera, las intervenciones realizadas en el área de la anestesiología. Al respecto, Marriner (1999) menciona que la enfermería en sus inicios se basó en el análisis, razonamiento y argumentación lógica de fenómenos propios, motivo por el que se le consideró más un arte que una ciencia, lo cual propició el surgimiento de la filosofía humanista que la caracteriza, reflejada por sus valores, metas y opiniones en cuanto a su campo de acción, el cuidado de las personas, que en este caso se refiere a aquellas sometidas a proceso quirúrgico. 


\section{Revista Electrónica Enfermeria Actual en costa Rica}

De igual manera, cabe mencionar que esa base filosófica y epistemológica permitió el análisis de teorías de la enfermería clínica (Shafer, 1987), las cuales más tarde configurarían el conocimiento de la disciplina mediante teorías verificadas mediante la prueba empírica. Por lo anterior, a mediados del siglo XIX, Florence Nightingale enunció los primeros postulados que cimentaron la ciencia enfermera (Caballero, Becerra y Hullin, 2010) y desde los que se dilucidó y definió tanto el cuidado, como su esencia, representado por Colliere (1993) como una infinita variedad de actividades dirigidas a mantener y conservar la vida. Lo cual fue aplicado en el quehacer cotidiano de la enfermería en anestesia donde se condensa todo este cuidado y conocimiento que se empezaba a cimentar en Costa Rica.

Lo anterior dilucida diversas transformaciones de la enfermería en diferentes contextos históricos que impulsaron el desarrollo de una práctica basada en un conocimiento propio, intrínsecamente diferente a las otras ciencias médicas (Marriner, 1999), con fundamento en principios determinados por la formación basada en el aprendizaje desarrollado por años de experiencia (Wesley,1997).

Lo mencionado anteriormente, justificó el planteamiento del objetivo de esta revisión histórica, que fue identificar las contribuciones de la enfermería a la anestesiología en Costa Rica.

\section{MATERIALES Y MÉTODO}

Las personas participantes del estudio fueron 23 personas, 12 mujeres y 11 varones, de las que 5 mujeres y 5 hombres ya estaban pensionados con más de 15 años de laborar en anestesia. El resto es personal activo que labora en hospitales nacionales.

Se aplicó la metodología recomendada para llevar a cabo investigación histórica que estudia hechos ocurridos al ser humano en épocas pasadas (Peréx, 2013), razón por la que se analizó libros, documentos y datos recolectados en entrevistas y en grupo focal a profesionales de la disciplina, activos y pensionados, que tuvieron relación directa con la anestesiología en Costa Rica.

Para lograrlo se llevó a cabo las siguientes fases de la investigación histórica propuestos por Peréx (2013): en la fase preliminar, se formuló una hipótesis. Luego, se elaboraron los antecedentes de la temática mediante el análisis de documentos escritos y fuentes primarias y secundarias. Posteriormente, se pasó a la segunda fase heurística de recopilación y sistematización de fuentes, clasificada en primarias y secundarias mediante el uso fichas. En la tercera, la fase hermenéutica, se valoró críticamente las fuentes primarias y secundarias para llegar a la última fase, la ensayística, con la que se ordenó las fuentes y se construyó la síntesis.

En primera instancia se revisó la base de datos del Colegio de Enfermeras para identificar el número de profesionales en enfermería con la especialidad en anestesiología y establecer los primeros contactos. Sin embargo, en dicha institución no se encontró datos ya que se inscribían solo como profesionales en enfermería. Por lo anterior, se consultó al Colegio de Médicos de Costa Rica dónde se pudo constatar que en el año 2009 había 121 enfermeras y enfermeros anestesistas inscritos, de los cuales 94 laboraron para la Caja Costarricense de Seguro Social (CCSS). Luego, en año 2000, solo se encontraban activas 47 y en el 2010, quedaron 23 inscritas y 


\section{Revista Electrónica Enfermeria Actual en costa Rica}

activas, los cuales fueron los participantes del estudio. Se contactó a las personas de forma personal, vía telefónica y por fax, para la realización del grupo focal y entrevistas individuales.

Las fuentes orales, según Meihy (1996), se refieren al relato oral desde la perspectiva de las personas que experimentaron y contribuyeron en el proceso histórico, que en este caso correspondió a profesionales en enfermería certificados como técnicos en anestesia y que estuvieron directamente relacionados en términos de lugares y tiempo. A estas personas se les aplicó la entrevista y el grupo focal. También fueron utilizadas otras fuentes de información como documentos escritos de salas de operaciones, de archivos de hospitales y libros como la Reseña de la anestesia en Costa Rica, escrita por el doctor Sotela en 1997.

La información recolectada se trianguló utilizando diferentes fuentes y métodos, con el propósito de organizar las unidades categoriales (Willing, 2008), mencionado por Hernández, Fernández y Baptista (2010), describir experiencias de las personas (Creswell, 2009) mencionado por Hernández et al (2010), y comprender el contexto.

En cuanto al proceso de análisis, se realizó mediante los tres niveles presentados por Peréx (2013), los cuales se detalla a continuación: en el primer nivel, se elaboró fichas para recuperar la información clasificada según fuentes primarias y fuentes secundarias, las cuales contienen información como nombre, año, lugar de hechos, así como citas textuales (Hernández et al, 2010). En el segundo, se construyó tablas que incluían la secuencia de contenido y el análisis conceptual. Finalmente, en el tercero se categorizó según momentos históricos y sus características.

Del proceso anterior surgieron las siguientes dimensiones y categorías de análisis:

\section{Dimensión: Inicios de la anestesia en Costa Rica}

\section{1.a Categorías: inicios de la anestesiología y personas protagónicas}

2. Dimensión: Preparación formal en anestesia

2.a Categorías: impacto y labor desempeñada por la enfermería

La confiabilidad de los resultados es garantizada por el análisis crítico y acercamiento oportuno, meticuloso, cuidadoso y respetuoso a las fuentes primarias y secundarias. Debido a la importancia del estudio, se sometió a prueba la confiabilidad, la cual Peréx (2013) sitúa como característica determinante; para lograrlo se cotejó las condiciones en que se dieron los hechos en la historia y se estableció relaciones, entre los datos de las fichas y los que llevaron a categorizar los resultados.

\section{Consideraciones éticas}

Se cumplió con el principio de autonomía, que garantiza a las personas la completa potestad de tomar decisiones sobre su participación durante el desarrollo del estudio, entre las que se incluye la capacidad de comprender el porqué y para qué de este, además de que expresan su voluntad para participar o no participar y establecer criterios en condiciones de libertad, sin influencia o presión por parte de la investigadora; es decir, la participación es completamente voluntaria. Para lograr dicho principio se les solicitó su consentimiento 


\section{Revista Electrónica Enfermeria Actual en costa Rica}

informado, en el que se puntualiza el objetivo de la investigación, la manera que se utilizará la información, su carácter confidencial y todas aquellas intervenciones referentes a su participación en todos los ámbitos.

Otros principios éticos aplicados son el de no maleficencia para la persona y la organización donde laboran, sin generarles consecuencias negativas, ya que se guarda confidencialidad y se asegura que no haya implicaciones legales, de salud, sociales o políticas. De igual manera se cumplió con el de beneficencia, puesto que todas las actividades desarrolladas son para beneficiar a las personas involucradas y a las organizaciones. Finalmente, el principio de justicia se logró mediante el trato equitativo brindado a las personas participantes sin importar edad, género u otras condiciones.

\section{RESULTADOS}

Entre los resultados del estudio se destaca que, durante más de 90 años, el personal de enfermería suministró anestesia a personas sometidas a un proceso quirúrgico (Sotela, 1997), función realizada tanto en hospitales del área metropolitana, como rurales. Sin embargo, en 1981 se suspendió la preparación de técnicos y técnicas de anestesia, aluciéndose que era una etapa superada.

\section{Dimensión: Inicios de la anestesia en Costa Rica}

Categoría: Inicios de la anestesia en Costa Rica y personas protagónica.

Los inicios de la anestesia en Costa Rica se remontan a 1875, de la mano de religiosas que practicaban la enfermería en el hospital San Juan de Dios, quienes fueron instruidas por el doctor Carlos Durán en la técnica de administrar anestesia que consistía en administrar cloroformo "gota a gota", a través de una mascarilla facial de alambre, forrada con ocho capas de gasa, así lo indica Sotela (1997) al detallar que

cuando llegaron los primeros frascos de cloroformo el doctor Durán les mostró la forma de administrarlo. A partir de ahí y hasta 1965, durante 90 años continuaron dando las anestesias en diferentes hospitales del país, sobre todo en aquellos más alejados de la capital (p. 22).

De este modo las religiosas que practicaban la enfermería fueron protagonistas al convertirse en unas de las primeras personas en Costa Rica en administrar anestesia a individuos sometidos a procesos quirúrgicos, lo cual marcó un hito en la historia de la anestesia y en la salud pública del país, pues, entre otros, contribuyó a disminuir la mortalidad quirúrgica del país, hecho confirmado por González (1995), quien añade que "del bienio 1920-1921 fue de 2,22\%, del bienio 1922-1923 fue de 1,72\%, del bienio 1924- 1925 fue de 1,12\%, del bienio 1926-1927 de 1,30\%, 1930-1931 de 0,57\%" (p. 204).

Cabe resaltar que en Costa Rica, antes de 1942 no había médicos especializados en anestesiología, con título que los acreditara, solo la practicaban en caso necesario, pues el número de cirugías había aumentado en comparación con el número de enfermeras anestesistas, según consta en el Registro de las intervenciones quirúrgicas del Hospital San Juan de Dios, (p. 23), en el que se menciona que 


\section{Revista Electrónica Enfermeria Actual en costa Rica}

el Director del hospital San Juan de Dios de 1962 a 1976, cuando llegó de Bélgica, país en donde hizo sus estudios de medicina, tuvo que pasar algún tiempo "dando" anestesias antes de que le permitieran realizar su primera operación, (Sotela, 1997, p. 21).

Como hemos visto antes de 1940 nadie imaginaba en Costa Rica que la anestesiología pudiera llegar a ser una especialidad médica pues los primeros 25 años (de 1875 a 1900) fue exclusivamente de Enfermería (Sotela, 1997, p. 47)

Ahora bien la participación del profesional de enfermería en anestesia es interpretado por quienes la practican de la siguiente forma

Una enfermera de anestesia, participa en la realización de técnicas avanzadas y especializadas, a pacientes que requieran de los cuidados, respiratorios, resucitación cardiopulmonar u otras emergencias o servicios de mantenimiento de la vida. (Participante 3 grupo focal).

En Costa Rica, dada la significancia en la labor de atención a la persona sometida a un proceso quirúrgico, el doctor Sotela quiso darles un lugar en la historia, al consignar algunos de sus nombres.

Como homenaje y justo reconocimiento a los años de abnegada y eficiente labor, quiero consignar sus nombres: Dora Acuña, Etelvira Acuña, Rodrigo Alfaro, Vilma Argueas, Lilian Aragón, Llezenia Bonolla, Maruja, Marta Hidalgo, Gladiz León, Jorge Monge, Mercedes Morales, Miriam Murillo,Elena Rojas, José Sánchez, Lilibeth Varela, Norma Vega, Esperanza Villalobos y Margarita Zúñiga, (Sotela, 1997, pp. 82-83).

\section{Dimensión: Preparación formal}

Categoría: impacto y labor desempeñada

En el año 1956 la Escuela de Enfermería y la Dirección del Hospital San Juan de Dios, en la persona del doctor Sotela y otros médicos, capacitó formalmente al personal de Enfermería que laboraba en esta área. El entrenamiento era intensivo: se prolongaba duraba seis meses y los participantes eran acreditados como auxiliares de anestesia, lo cual quedó documentado por Sotela (1997), quien cuenta que

el doctor Sotela inició en el San Juan de Dios en 1956 una serie de cursillos patrocinados por la Escuela de Enfermería y la Dirección del Hospital, a fin de capacitar. La idea era darles conocimientos de farmacología, de fisiología de técnica de intubación, pero sobre todo enseñarles a manejar las máquinas de anestesia (p.77).

A partir de dicho momento se evidencia también que la segunda preparación fue en 1971, puesto que, por el impacto de la labor desempeñada por Enfermería en el área de la anestesiología, en 1971 el doctor Luis Guillermo Hidalgo, asistente del Servicio de Anestesia en el Hospital San Juan de Dios, desarrolló un programa para formar técnicos en anestesia, labor llevada a cabo por el Programa de Tecnologías en Salud, de la Escuela de 


\section{Revista Electrónica Enfermeria Actual en costa Rica}

Medicina de la Universidad de Costa Rica (Sotela, 1997). En este sentido una de estas enfermeras formadas en este programa de técnicos en anestesia refiere

Fuimos llamadas para capacitarnos en anestesia, no solo para trabajar en hospitales del área metropolitana, sino en rurales como el de Puntarenas. (Participante 1, grupo focal).

El programa solicitaba un requisito primordial, ser enfermera o enfermero profesional, además de una duración de año y medio, periodo en el que se incluía clases teóricas y prácticas, con temas diversos como el manejo de máquinas de anestesia, anatomía y fisiología de los diferentes sistemas del organismo, farmacología y diferentes técnicas de intubación, entre otros. Sin embargo, a pesar de la importante labor de estas personas, había inquietud respecto de la preparación de los técnicos.

No faltaron personas que nos criticaron por preparar técnicos, un colega dijo que por culpa de los doctores Sotela e Hidalgo el país está lleno de técnicos (Sotela, 1997, p. 80).

Por otro lado, cabe resaltar que en esa época la Escuela de Medicina de la Universidad de Costa Rica no había iniciado el posgrado en anestesia, sino hasta 1974, año en que se creó la Cátedra de Anestesiología.

Por otro lado, las y los enfermeros anestesistas reconocen los motivos que originaron el desarrollo de su carrera, y la importancia de ese momento histórico, dado por la falta de especialistas, situación que aún hoy en día se presenta. En este sentido es importante resaltar que es hasta 1976 que se gradúa la primera promoción de médicos anestesiólogos en esta línea el Sotela (1997) refiere que

En 1976 (primera promoción), en la Universidad de Costa Rica se graduaron 7 anestesiólogos. Dos más en el 77, cuatro en el 78 y uno en el 79. Para 1981 se suspendieron los cursos para Técnicos en Anestesia. (p. 81).

Sin embargo, a pesar de los pocos médicos graduados en anestesiología se estimó que la etapa estaba superada y el propósito se había cumplido, por lo que se cerró el programa de técnicos en anestesia (Sotela, 1997). A pesar de las situaciones mencionadas, hace sentir a estas personas, gran satisfacción en su labor cumplida, así lo constata el participante 1, al mencionar que "hemos aportado a la atención de las personas, es suficiente para nosotros, además de que durante todos estos años nuestra labor ha sido reconocida por las autoridades de la Caja Costarricense de Seguro Social."

El contexto histórico en el que surgieron las situaciones mencionadas permitió conectar a personas, instituciones y acontecimientos e interpretar vivencias que las llevaron a ver la necesidad de aumentar su conocimiento, tal como agrega la participante 2: "Tenemos un trabajo muy especializado sabemos que la persona que atendemos depende completamente de nosotros eso nos anima a estar al día, más que todo en materia de anestésicos y en técnicas quirúrgicas."

A pesar de que los enfermeros técnicos en anestesia consideran que su labor es y ha sido muy especializada, por el cuidado minucioso que requieren las y los usuarios durante el proceso quirúrgico, en los últimos años quienes se mantienen activos ven cómo su labor se ha visto relegada, realidad que les produce 


\section{Revista Electrónica Enfermeria Actual en costa Rica}

frustración y por la que consideran dotar la enfermería de posgrados que la sitúen en el equipo de salud con un papel protagónico basado en perfiles profesionales acordes con su preparación.

\section{DISCUSIÓN}

En la historia de la enfermería concurren hechos acaecidos durante su desarrollo profesional, particularmente en el área de desempeño de la anestesiología, lo cual es imprescindible para comprender y vincular el presente y darle validez, labor que requirió tanto de documentos escritos como relatos de enfermeras y enfermeros involucrados en el contexto histórico, cuyos aportes confirman y destacan el papel protagónico de la disciplina enfermera.

Los resultados demuestran las contribuciones de la enfermería, no solo a la anestesia, sino a la salud en Costa Rica, puesto que durante más de 90 años fue una actividad propia de la disciplina, no realizada por otras. Es oportuno mencionar, que se eliminó sin justificación el que los y las enfermeras proporcionaran anestesia, de acuerdo con los libros de actas universitarias. Desde la óptica de la comunicación, se establecieron en ese momento relaciones de poder fáctico y efectivo (Faucol, 1999), esto por cuanto nunca se comentó o preguntó a enfermería sobre el porqué y el cómo se eliminó la preparación formal de Técnicos (as) en anestesia. De igual manera, otra situación de manejo del poder es el no permitir el análisis de opiniones de los profesionales respecto del saber propio y del generado en esta área: simplemente se tomó la decisión de eliminarla por considerarla derecho de la profesión médica (Faucol, 1999). Esta situación de aceptación del saber médico por parte de enfermería aun considerando que ya se contaba con saberes propios en ésta área, ha sido motivo de estudios y análisis por parte de diferentes epistemólogas de la disciplina, como Carper ( 1978) quien respecto del saber, hacer y ser de la enfermería identificó tipos de conocimiento: empírico, estético, personal, ético, sociopolítico, histórico.

En relación con el tema, también Kerouac (1996), Meleis (1987) y Meleis (1999) elaboraron un análisis epistemológico de los saberes de enfermería y los catalogaron como silencioso, adquirido, subjetivo y procedimiento construido, de los cuales se destacan el conocimiento silencioso, definido como la tendencia de enfermería a aceptar las voces de autoridad, aprendiendo a ser silenciosas y el conocimiento adquirido que es la creencia en la habilidad de la autoridad externa para generar conocimiento, pero no en sus propias habilidades para hacer lo mismo, pensando que es superior al propio.

Otro aspecto se refiere a las relaciones de poder entre las dos disciplinas (medicina y enfermería) que Faucol (1999) presenta desde la concepción jurídica, como un derecho y, desde la óptica de la comunicación, como un poder fáctico y efectivo que no se somete a escrutinio, ni siquiera -en este caso- al saber de la enfermería con conocimientos en anestesia, lo cual se debe a su descalificación por ser considerado no conceptual, insuficientemente elaborado o excluido del ámbito científico.

Ese juego de saberes motiva que el autor los tipifique como descalificados, por tanto anulados, todo en nombre de los derechos de la ciencia médica, de modo que se establece una relación fáctica entre poder, saber y verdad que da lugar a la ausencia en la búsqueda real y congruente entre saberes. Al respecto, se menciona el hecho histórico que Sotela (1997) describe como la eliminación de preparación de técnicos en anestesia y que coincide con las primeras promociones de médicos anestesistas graduados por la Universidad de Costa Rica. Los 


\section{Revista Electrónica Enfermeria Actual en costa Rica}

informes detallan que en 1977 se graduaron las primeras siete personas, en 1978, cuatro personas y en 1979, una fecha correspondiente a la última generación de enfermeros anestesistas, pues como el autor lo menciona ya se había cumplido el propósito de preparar a enfermería, aunque no señala dicho propósito, como una etapa superada, de manera que cerró su participación en la anestesia en Costa Rica.

Cabe mencionar que Enfermería como ciencia apoyada en un perfil profesional definido y basado en el cuidado integral y humanístico a la persona, familia y comunidades, en este caso durante los procesos quirúrgicos, es parte del equipo de salud que da respuesta a las necesidades de atención en salud, lo cual, a su vez, responde a la pregunta de la investigación sobre la contribución en el área de la anestesia en Costa Rica.

La enfermería en anestesia ha existido desde los inicios de la atención hospitalaria del país, como una necesidad de atención de la época y que aún presente en nuestros días. Su comprensión histórica lleva a conocer el interés que siempre ha existido, en su desarrollo y la participación de Enfermería en sus memorias. Su aportación en su progreso está impresa en los libros de actas de las salas de operaciones.

Las y los profesionales de enfermería en anestesia son reconocidos como actores sociales comprometidos, críticos y responsables en la atención de las personas sometidas a procesos quirúrgicos, labor llevada a cabo como profesionales en salud, con autonomía y capacidad de trabajo multidisciplinar que, como parte del equipo de salud y desde su enfoque disciplinar, se articula con las otras disciplinas para obtener el objetivo común: la recuperación de las personas (García, 1983). Al mismo tiempo, realizan un trabajo interdisciplinar porque al ser parte integral del equipo realiza una tarea concreta en común con sentido integral.

\section{CONCLUSIÓN}

La preparación formal de las enfermeras como técnicas en anestesia estuvo a cargo del doctor Sotela mediante un programa intensivo de seis meses, que acreditaba a las personas como auxiliares de anestesia, el cual, en 1971, fue incluido por la Escuela de Medicina de la Universidad de Costa Rica en el programa de Tecnologías en Salud, el de Técnicos en anestesia, con una duración de año y medio.

La historia evidencia que las contribuciones de la enfermería a la anestesiología en Costa Rica, al igual que en otras partes del mundo fueron importantes en la atención en salud, dado que su participación activa al prodigar cuidados integrales a la persona, familia y comunidad, no solo al disminuir índices de morbilidad sino también como recurso humano que se desplazó a áreas rurales, no fue suficiente motivo para continuar siendo parte del equipo de salud quirúrgico, puesto que fue suplantado por otros profesionales, con igual importancia en la atención pero con un perfil profesional diferente. A pesar de los aportes que han dado y siguen dando los profesionales de enfermería en esta área del saber, las autoridades en materia de salud consideran que no es necesario preparar a los y las enfermeras en anestesia ya que esta tarea es llevada a cabo por otros profesionales, respuesta que no satisface las necesidades de atención de la población en general. 


\section{Revista Electrónica Enfermeria Actual en costa Rica}

\section{REFERENCIAS BIBLIOGRÁFICAS}

Caballero R., Becerra y Hulim (2010) Proceso de enfermería e Informática para la gestión del cuidado. Santiago: Editorial Mediterráneo.

Carper. B. (1978). Fundamental patterns of Knowing in Nursing Science. Advances in Nursing Science, 1(1): 13-23

Colegio de Enfermeras de Costa Rica (2012). Libros de Actas.

Colegio de Médicos de Costa Rica (2012). Comité de tecnólogos de Costa Rica. Costa Rica. Departamento Legal, Costa Rica.

Colliere, M. (1993). Promover la Vida. New York: Mc Graw Hill.

Foucol, M. (1999). La arqueología del saber. Decimonovena edición. México D. F.: Siglo XXI. Editores S.A.

García, M. (1983). Trabajo en equipo. México D. F.: Ed. Fondo Interamericano.

González, C. (1995). Hospital San Juan de Dios, 150 años de Historia. San José: Editorial Nacional de Salud y Seguridad Social.

González, S., Mugabure, B., Uria, A. (2010) Actualizaciones en anestesiología para enfermería. Hospital Donostia. Gobierno Vasco: Osakidetsa. Recuperado de http://www.osakidetza.euskadi.eus

Hernández, Fernández y Baptista. (2010). Metodología de la investigación. México D. F.: Mc Graw Hill.

Higgins, LF. (s.f.) Cronohistoriografía de la anestesiología. Recuperado de:

http://www.anestesia.com.mx/histor2.html

Kerouac, Suzanne y otros (1996). El pensamiento enfermero. Madrid: Masson

Marriner, A. (1997). Modelos y teorías de Enfermería. Madrid: Harcourt Brace de España S.A.

Meihy, J. (1996). Historia oral: cómo hacer, cómo pensar. Universidad de Sao Paulo. Sao Paulo.

Meleis, A. (1987). Epistemology: The nature of knowledge. Boston: School of Nursing.

Meleis, A. (1999). Theorical Nursing: development and progress (3rd. ed.). Philadelphia: Lippincott-Raven.

Peréx, J. (2013). Métodos y técnicas de la investigación histórica. Madrid: Universidad Nacional de Educación a Distancia.

Schafer, P. (1987). Philosophic analysis of a theory of clinical nursing. Maternal Child Nursing Journal, 16 (4): 289368. 


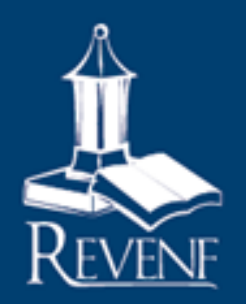

\section{Revista Electrónica Enfermeria Actual en costa Rica}

Sotela, J. (1997). Reseña Histórica de la Anestesia en Costa Rica. San José: Editorial Nacional de Salud y Seguridad Social.

Vélez, G. (1996) Desarrollo de la Enfermería en Anestesia en el continente americano. San Juan de Puerto Rico. Recuperado de: http://www.aseedar-td.org/revistas/articulos/06-4.pdf

Wesley, L. (1997) Teorías y Modelos de Enfermería. Segunda Edición. México D.F.: Mc Graw Hill. 